\title{
In vitro activity of immunosuppressive drugs against Plasmodium falciparum
}

\author{
Luzia Veletzky ${ }^{1}$, Khalid Rehman ${ }^{1}$, Tilman Lingscheid ${ }^{1,2}$, Wolfgang Poeppl ${ }^{1}$, Felix Loetsch ${ }^{1}$, Heinz Burgmann ${ }^{1}$ \\ and Michael Ramharter ${ }^{1,3^{*}}$
}

\begin{abstract}
Background: Solid organ transplant recipients are particularly vulnerable for infectious diseases due to prolonged immunosuppressive treatment. Residents of endemic regions and travellers may be exposed to malaria and may, therefore, require prolonged antimalarial chemoprophylaxis. The hypothesis of this study was that certain immunosuppressive drugs may exert clinically relevant anti-malarial activity. It was therefore designed to assess the intrinsic anti-malarial activity of everolimus, mycophenolic acid, and rapamycin against Plasmodium falciparum in an in vitro model.
\end{abstract}

Methods: Three laboratory adapted clones of $P$. falciparum and two isolates were used to assess the potential of mycophenolic acid, rapamycin and everolimus to inhibit in vitro growth of $P$. falciparum. The standard histidine rich protein 2 assay was employed and inhibitory drug concentrations (IC) were computed by non-linear regression analysis.

Results: All drugs were associated with complete inhibition of $P$. falciparum growth in in vitro assays. Mycophenolic acid demonstrated $I C_{50}$ and $I C_{90}$ values of $5.4 \mu \mathrm{mol} / \mathrm{L}$ and $15.3 \mu \mathrm{mol} / \mathrm{L}$. Rapamycin inhibited $P$. falciparum growth at $7.2 \mu \mathrm{mol} / \mathrm{L}\left(\mathrm{IC}_{50}\right)$ and $12.5 \mu \mathrm{mol} / \mathrm{L}\left(\mathrm{I} \mathrm{C}_{90}\right)$, respectively. Finally, everolimus displayed $I C_{50}$ and $I C_{90}$ values of 6.2 $\mu \mathrm{mol} / \mathrm{L}$ and $11.5 \mu \mathrm{mol} / \mathrm{L}$. There was no difference in in vitro activity against chloroquine sensitive or chloroquine resistant parasites.

Conclusions: All immunosuppressive drugs evaluated in this in vitro study demonstrated activity against P. falciparum. Inhibitory concentrations of mycophenolic acid are within clinically achievable plasma concentrations when used in solid organ transplant recipients. Further in vivo evaluation of mycophenolic acid either alone or in combination regimens may prove promising for the concomitant prevention of $P$. falciparum in solid organ transplant recipients living or travelling in malaria endemic regions.

Keywords: Plasmodium falciparum, Immunosuppressives, Mycophenolic acid, Rapamycin, Everolimus

\section{Background}

Despite international efforts to control and ultimately eliminate malaria, Plasmodium falciparum remains among the three most deadly infectious pathogens in sub-Saharan Africa causing an estimated 627,000 deaths every year [1]. One of the most formidable challenges in the fight against falciparum malaria is the ongoing emergence and spread of drug resistant isolates leading to reduced efficacy and effectiveness of established anti-malarial therapies [2]. Large-

\footnotetext{
* Correspondence: michael.ramharter@medizin.uni-tuebingen.de 'Department of Medicine I, Division of Infectious Diseases and Tropical Medicine, Medical University of Vienna, Währinger Gürtel 18-20, 1090 Vienna, Austria

${ }^{3}$ Institut für Tropenmedizin, Universität Tübingen, Tübingen, Germany Full list of author information is available at the end of the article
}

scale in vitro screening of drug candidates is performed to discover new compounds with anti-malarial activity [3]. However, only a fraction of those with proven in vitro activity are successfully developed to a marketed drug mostly due to concerns with safety and tolerability in preclinical or clinical development. Screening of drugs already used in clinical practice for other indications than malaria is another valuable approach to build-up the anti-malarial armamentarium. Drugs which are used for chronic conditions necessitating lifelong treatment may be of particular interest for their use as anti-malarial prophylactic agents.

The ever growing number of patients requiring immunosuppressive therapy after solid organ transplantation is particularly vulnerable for infectious diseases. Whereas 
rapamycin - commonly referred to as sirolimus - and cyclosporine A have been shown to exhibit anti-malarial activity in vitro, no conclusive data are available for everolimus and mycophenolic acid - two of the most widely used immunosuppressive drugs [4]. An immunosuppressive combination treatment with collateral prophylactic efficacy against malaria would constitute an important benefit for solid organ transplant recipients in malaria endemic regions and travellers to tropical countries. This would be of even higher clinical benefit as immunosuppressive drugs are prone to clinically significant drug interactions and the interaction of anti-malarials and immunosuppressive drugs is currently not well understood. This study was, therefore, designed to assess the in vitro activity of mycophenolic acid, everolimus and rapamycin against $P$. falciparum to obtain further information about the potential of currently used immunosuppressives as anti-malarial prophylactic drugs.

\section{Methods}

\section{Laboratory strains and clones}

The study was performed with three $P$. falciparum clones with distinct anti-malarial resistance patterns (Dd2, 3D7 and 7G8) and two laboratory-adapted strains from returning travellers. Clones Dd2 and 7G8 are resistant against chloroquine, whereas 3D7 sensitive to all commonly used anti-malarials [5]. Laboratory adapted strains originated from two returning travellers who acquired $P$. falciparum infections in West Africa. These strains demonstrate high level resistance against chloroquine but are sensitive against mefloquine and artemisinin derivatives. Parasites were kept in continuous malaria culture as described previously [6].

\section{Test compounds}

Chloroquine diphosphate salt (Sigma, C6628) was dissolved in double distilled water. Mycophenolic acid (Fluka, 70018), rapamycin (Sigma, R0395) and everolimus (Fluka, 07741) were dissolved in absolute ethanol. All stock solutions were further diluted in double distilled water to obtain respective target concentrations. 96-well culture plates were coated with ascending concentrations of chloroquine $(0.003-3 \mu \mathrm{mol} / \mathrm{L})$, mycophenolic acid (0.6$39 \mu \mathrm{mol} / \mathrm{L})$, rapamycin $(0.2-13.7 \mu \mathrm{mol} / \mathrm{L})$ and everolimus $(0.2-13 \mu \mathrm{mol} / \mathrm{L})$. Test plates were dried and kept at $4{ }^{\circ} \mathrm{C}$ for a maximum of two weeks before use. All drug assays were performed in duplicate with all $P$. falciparum clones and strains.

\section{Drug sensitivity assay}

Cultured parasites were incubated for 72 hours on precoated plates and drug sensitivity assays were performed using an ELISA for quantitative measurement of the P. falciparum specific histidine rich protein 2 (HRP-2). ELISA plates were coated with commercial monoclonal antibodies and tests were performed as previously described [7]. An at least four-fold increase in HRP-2 concentrations was set as threshold for further statistical analysis of drug sensitivity assays. Statistical analysis was performed and inhibitory concentrations (IC) were calculated using freely available software [8]. This program was developed to analyse data from malarial drug sensitivity tests such as the HRP-2 assay and produces non-linear regression models to calculate individual inhibitory concentrations (IC). No ethical clearance was required for this in vitro study following regulations of the Ethics Committee of the Medical University of Vienna.

\section{Results}

Immunosuppressives

All drugs were tested in parallel against the three clones and two strains of $P$. falciparum resulting in 20 evaluable drug-concentration/growth-inhibition curves. Growth rates were sufficient in all assays for further analysis. Mycophenolic acid led to complete inhibition of in vitro growth of $P$. falciparum at $19.5 \mu \mathrm{mol} / \mathrm{L}$ and $39 \mu \mathrm{mol} / \mathrm{l}$, respectively. Complete growth inhibition was achieved at highest concentrations of rapamycin $(13.7 \mu \mathrm{mol} / \mathrm{L})$ and at $13 \mu \mathrm{mol} / \mathrm{L}$ of everolimus for all tested clones and isolates. Individual 50 and 90 percent inhibitory concentrations are shown in Table 1.

Table 1 Individual IC 50 and IC 9 values for respective immunosuppressive drugs are shown for each tested parasite sample and for chloroquine resistant strains, respectively

\begin{tabular}{|c|c|c|c|c|c|c|c|c|c|c|c|c|}
\hline \multicolumn{11}{|c|}{ Individual $I C_{50}$ and $I C_{90}$ values $^{1}$} & \multirow{2}{*}{\multicolumn{2}{|c|}{$\begin{array}{c}\text { Median } \mathrm{IC}_{50} \text { and } \mathrm{IC}_{90} \text { values } \\
\mathrm{CQr}^{2} \text { parasites }\end{array}$}} \\
\hline & \multicolumn{2}{|c|}{$3 d 7$} & \multicolumn{2}{|c|}{ 7G8 } & \multicolumn{2}{|c|}{ Dd2 } & \multicolumn{2}{|c|}{ Strain 1} & \multicolumn{2}{|c|}{ Strain 2} & & \\
\hline & $\mathrm{IC}_{50}$ & $\mathrm{IC}_{90}$ & $\mathrm{IC}_{50}$ & $I C_{90}$ & $\mathrm{IC}_{50}$ & $\mathrm{IC}_{90}$ & $\mathrm{IC}_{50}$ & $\mathrm{IC}_{90}$ & $\mathrm{IC}_{50}$ & $\mathrm{IC}_{90}$ & $\mathrm{IC}_{50}$ & $I_{90}$ \\
\hline Mycophenolic acid & 5.3 & 15.1 & 5.4 & 15.3 & 5.7 & 16.7 & 5.4 & 14.4 & 6.4 & 23.7 & $5.6(5.4-5.9)^{3}$ & $16(15.1-18.5)$ \\
\hline Rapamycin & 8.1 & 12.5 & 6.7 & 11.8 & 6.6 & 12.3 & 7.2 & 12.7 & 7.8 & 12.7 & $6.9(6.6-7.4)$ & $12.5(12.2-12.7)$ \\
\hline Everolimus & 7.5 & 11.8 & 5.1 & 10.7 & 6.4 & 11.5 & 5.8 & 11.2 & 6.2 & 11.6 & $6(5.7-6.3)$ & $11.3(11.1-11.5)$ \\
\hline Chloroquine & 0.01 & 0.03 & 0.19 & 1.05 & 0.22 & 1.09 & 0.21 & 0.65 & 0.27 & 0.73 & $0.21(0.2-0.23)$ & $0.9(0.7-1.1)$ \\
\hline
\end{tabular}

1 in $\mu \mathrm{mol} / \mathrm{L}, 2$ Chloroquine resistant, 3 interquartile range. 


\section{Evaluation of cross-sensitivity between chloroquine and immunosuppressive drugs}

As shown by the individual IC values in Table 1, 3D7 was the only chloroquine sensitive parasite tested in this trial. Median results for chloroquine resistant parasites are presented in comparison and no consistent pattern of alterations in anti-malarial activity of immunosuppressive drugs in chloroquine resistant versus chloroquine sensitive parasite samples was observed.

\section{Discussion}

Mycophenolic acid, rapamycin and everolimus reliably inhibited in vitro growth of P. falciparum in our study. Inhibition was independent of chloroquine susceptibility and was observed in all tested parasites. Therapeutic target concentrations are in the range of around 5.4-32.8 nmol/L for rapamycin and 3-15.7 nmol/L for everolimus when used as immunosuppressive drugs after solid organ transplantations [9]. As the in vitro growth inhibition is at considerably higher concentrations, these drugs are unlikely to exert clinically important anti-malarial activity in vivo. On the contrary, inhibitory concentrations for mycophenolic acid were within clinically relevant plasma levels in patients using standard immunosuppressive regimens for solid organ transplantation. Peak plasma levels after oral intake of $1 \mathrm{~g}$ mycophenolic acid are around $78 \mu \mathrm{mol} / \mathrm{L}$ [10]. Importantly, mycophenolic acid has a relatively long half-life of around 17 hours leading to sustained high plasma concentrations in currently recommended twice oral daily regimens. Trough levels under steady state conditions are around $10 \mu \mathrm{mol} / \mathrm{L}$ indicating the potential for prolonged anti-malarial in vitro activity of mycophenolic acid [11]. With all caveats applicable for extrapolation of in vitro data to the in vivo situation, these results clearly demonstrate the potential of clinically important activity of mycophenolic acid against $P$. falciparum. Although this drug is unlikely to be used as a targeted treatment for malaria, its clinical usefulness as prophylactic agent in transplant recipients may be further investigated. It may be speculated that the use of mycophenolic acid alone - or in combination with other immunosuppressive drugs - may provide sufficient blood schizontocidal activity to preclude the need for additional chemoprophylaxis.

Interestingly, there is a historic link of anti-malarials and immunosuppressive drugs with the 4-aminoquinolines as the most prominent example. Chloroquine - first developed as anti-malarial drug - has known immunomodulatory effects and is used on a large scale for the treatment of rheumatic diseases including lupus erythematosus [12]. Similarly, cyclosporine A and FK506 possess antiparasitic activity and have been considered as lead agents for new classes of antimicrobial drugs [13]. Mycophenolic acid may, therefore, become yet another example of an immunosuppressive drug with potentially clinically important anti-malarial activity.

\section{Competing interests}

The authors declare that they have no competing interests. MR is serving in the Editorial Board of the Malaria Journal.

\section{Authors' contributions}

LV contributed to the conception and design of the study, performed parasite cultivation, microscopic assessment and HRP-2 immunoassay, analysed the data and participated in drafting the manuscript. KR, TL, WP, FL, HB performed parasite cultivation, microscopic assessment, HRP-2 immunoassay and contributed to the conception and design of the study. MR conceived and designed the study, contributed to analysis of data, and drafted and revised the manuscript. All authors read and approved the final version of the manuscript.

\section{Acknowledgments}

The authors thank Heide-Maria Winkler from the Department of Interna Medicine I, Division of Infectious Diseases and Tropical Medicine, Medical University of Vienna for valuable suggestions, excellent technical assistance and support in the analysis of results.

\section{Funding}

No external support was obtained for this study. Costs were supported by departmental research funding by the Medical University of Vienna. We acknowledge support by Deutsche Forschungsgemeinschaft (DFG-Projekt KR 1150/6-1) and the Open Access Publishing fund of the University of Tübingen.

\section{Author details}

'Department of Medicine I, Division of Infectious Diseases and Tropical Medicine, Medical University of Vienna, Währinger Gürtel 18-20, 1090 Vienna, Austria. ${ }^{2}$ Department of Infectious Diseases and Respiratory Medicine, Charité Universitätsmedizin, Berlin, Germany. ${ }^{3}$ Institut für Tropenmedizin, Universität Tübingen, Tübingen, Germany

Received: 11 November 2014 Accepted: 27 November 2014 Published: 4 December 2014

\section{References}

1. WHO: World Malaria Report 2013. Geneva: World Health Organization; 2013.

2. Miotto O, Almagro-Garcia J, Manske M, Macinnis B, Campino S, Rockett KA, Amaratunga C, Lim P, Suon S, Sreng S, Anderson JM, Duong S, Nguon C, Chuor CM, Saunders D, Se Y, Lon C, Fukuda MM, Amenga-Etego L, Hodgson AVO, Asoala V, Imwong M, Takala-Harrison S, Nosten F, Su X-Z, Ringwald P, Ariey F, Dolecek C, Hien TT, Boni MF, et al: Multiple populations of artemisinin-resistant Plasmodium falciparum in Cambodia. Nat Genet 2013, 45:648-655.

3. Ramharter M, Noedl H, Thimasarn K, Wiedermann G, Wernsdorfer G, Wernsdorfer WH: In vitro activity of tafenoquine alone and in combination with artemisinin against Plasmodium falciparum. Am J Trop Med Hyg 2002, 67:39-43.

4. Bell A, Wernli B, Franklin RM: Roles of peptidyl-prolyl cis-trans isomerase and calcineurin in the mechanisms of antimalarial action of cyclosporin A, FK506, and rapamycin. Biochem Pharmacol 1994, 48:495-503.

5. Ramharter M, Wernsdorfer WH, Kremsner PG: In vitro activity of quinolines against Plasmodium falciparum in Gabon. Acta Trop 2004, 90:55-60.

6. Ramharter $M$, Noedl $H$, Winkler $H$, Graninger W, Wernsdorfer WH, Kremsner PG, Winkler S: In vitro activity and interaction of clindamycin combined with dihydroartemisinin against Plasmodium falciparum. Antimicrob Agents Chemother 2003, 47:3494-3499.

7. Noedl H, Bronnert J, Yingyuen K, Attlmayr B, Kollaritsch H, Fukuda M: Simple histidine-rich protein 2 double-site sandwich enzyme-linked immunosorbent assay for use in malaria drug sensitivity testing. Antimicrob Agents Chemother 2005, 49:3575-3577.

8. Malaria Drug Sensitivity Testing; http://malaria.farch.net [http://www.meduniwien.ac.at/user/harald.noedl/malaria/]

9. Mahalati K, Kahan BD: Clinical pharmacokinetics of sirolimus. Clin Pharmacokinet 2001, 40:573-585.

10. Bullingham RE, Nicholls AJ, Kamm BR: Clinical pharmacokinetics of mycophenolate mofetil. Clin Pharmacokinet 1998, 34:429-455. 
11. Staatz CE, Tett SE: Clinical pharmacokinetics and pharmacodynamics of mycophenolate in solid organ transplant recipients. Clin Pharmacokinet 2007, 46:13-58.

12. Ruiz-Irastorza G, Ramos-Casals M, Brito-Zeron P, Khamashta MA: Clinical efficacy and side effects of antimalarials in systemic lupus erythematosus: a systematic review. Ann Rheum Dis 2010, 69:20-28.

13. High KP: The antimicrobial activities of cyclosporine, FK506, and rapamycin. Transplantation 1994, 57:1689-1700.

doi:10.1186/1475-2875-13-476

Cite this article as: Veletzky et al: In vitro activity of immunosuppressive drugs against Plasmodium falciparum. Malaria Journal 2014 13:476.

\section{Submit your next manuscript to BioMed Central and take full advantage of:}

- Convenient online submission

- Thorough peer review

- No space constraints or color figure charges

- Immediate publication on acceptance

- Inclusion in PubMed, CAS, Scopus and Google Scholar

- Research which is freely available for redistribution 